\title{
Coexistence of Bifid Uvula and Cardiac Manifestations in a Patient: Is it a Syndrome or Coincidence?
}

\author{
Zeki Yüksel Günaydın ${ }^{1}$, Turgay Işık ${ }^{2}$, Osman Bektaş ${ }^{1}$ Erkan Ayhan ${ }^{2}$ \\ ${ }^{1}$ Ordu University Medical School, Department of Cardiology, Ordu, Turkey \\ ${ }^{2}$ Balıkesir University Medical School, Department of Cardiology, Balıkesir, Turkey
}

Received: 14. June 2015 Accepted: 8 August 2015/ published online: 30 August 2015

(C) Ordu University Institute of Health Science, Turkey, 2015

\begin{abstract}
Loeys-Dietz syndrome (LDS) is a rare life-threatening condition, with autosomal-dominant inheritance, which is caused by heterozygous mutations in the genes encoding transforming growth factor beta receptors 1 and 2 . It is characterized by a triad of tortuous arteries and aortic aneurysms, cleft palate or bifid uvula, and hypertelorism. Loeys-Dietz syndrome is a recently-described connective tissue disorder with features similar to those of Marfan syndrome, and the vascular type of Ehlers-Danlos syndrome. In Loeys-Dietz syndrome, the aortic aneurysms are prone to rupture at a smaller size than other aneurysms, putting children with Loeys-Dietz at great risk for dying if the aneurysm is not identified and treated early. Here we report 54-year-old-man who had atrial septal defect, aortic root dilatation and bifid uvula, which may be a variant of LDS.
\end{abstract}

Key Words: loeys-dietz syndrome, bifid uvula, atrial septal defect

\section{To the editor}

A 53 year-old man was admitted to the cardiology department with exertional dyspnea and atypical chest pain for 2 days. The patient reported no previous cardiac history except hypertension and diabetes mellitus. He had been used Ramipril $5 \mathrm{mg}$ orally once a day for 7 years and metformin $500 \mathrm{mg}$ orally twice a day for 5 years. On physical examination his blood pressure was $140 / 85 \mathrm{~mm} / \mathrm{hg}$ and heart rate was $72 \mathrm{bpm}$. In detail examination we detected the bifid uvula which was operated before. His father was operated due to bifid uvula in his family history (Figure).

Address for correspondence/reprints:

Zeki Yüksel Günaydın

E mail: doktorzeki28@ gmail.com

Phone num: +904522232426

DOI: $10.19127 / \mathrm{mbsjohs.21708}$

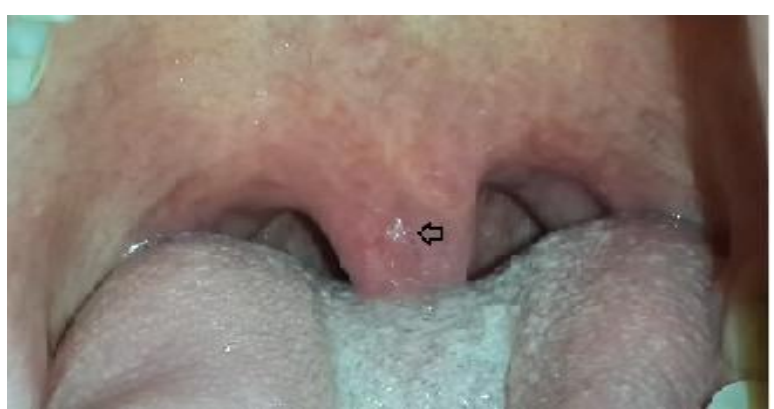

Figure 1: Bifid uvula which was operated before.

A 3/6 systolic murmur was heard in the pulmonary area on heart auscultation. The electrocardiography was sinus rhythm and there was no ischemic st and $t$ wave abnormalities.

Transthoracic echocardiography (TTE) showed dilated aortic root with $3.2 \mathrm{~cm}$, mild aortic valve regurgitation and a normal size of ascendan aorta. TTE also demostrated dilated right chambers and an abnormal turbulent flow along the interatrial septum that associated with an atrial septal defect. Transesophageal echocardiography showed the defect diameter of atrial septal defect was $1.4 \mathrm{~cm}$. There was also 
mild aortic regurgitation with bicuspid aortic valves. On computed tomography asendan, desendan, thoracic and abdominal aorta were in normal size. After the patient's consent the atrial septal defect closed with device procedure. The patient was discharged on medical treatment with 6 months acetylsalicylic acid and mentioned above hypertension and dibetes medication. In the postoperative follow-up the patient was asymptomatic and did not have any complication and was discharged in good condition.

The classic triad of Loeys-Dietz syndrome involves arterial tortuosity and aneurysms, hypertelorism, and bifid uvula or cleft palate (Loeys BL et al., 2006). Loeys-Dietz syndrome (LDS) resembles to Marfan syndrome, however it tends to be more aggressive. LDS have some difference from marfan syndrome such as lens dislocation etc. Also aortic root surgery should be considered with milder aortic root dilation (40 $\mathrm{mm}$ for Loeys-Dietz, versus $50 \mathrm{~mm}$ for Marfan) due to increased risk for aortic dissection and rupture with smaller aortic diameters (Williams JA et al., 2007). In initial reports, LDS patients were classified into two types depending on severity of craniofacial features (type 1) or cutaneous features (type 2) (Loeys BL et al., 2006). Some genetic mutations seen in LDS. Patients with mutations in TGFBR1, TGFBR2, SMAD3, or TGFB2 show more widespread and/or aggressive vascular disease when compared with Marfan syndrome or thoracic aortic aneurysm and dissection, irrespective of the severity of systemic features, a mutation in any of these genes in combination with documented aneurysm or dissection should be sufficient for the diagnosis of LDS. This will alert clinicians caring for these patients to the need for specialized patient counseling and management. At the same time the evidence based expansion of the clinical spectrum in LDS including patients with minimal or no dysmorphic features should be considered by clinicians. Such reasoning and practices have proven productive in the diagnosis and care of patients with Marfan and vascular Ehlers-Danlos syndromes (MacCarrick et al., 2014).

In our knowlegde coexistence of only bifid uvula and cardiac manifestations (atrial septal defect, bicuspid aorta, aortic root dilatation) have not been reported in literature before. However, these pathologies can be found together in Loeys-Dietz syndrome (LDS), which is an autosomal-dominant connective tissue disorder first characterized by aortic aneurysms and generalized arterial tortuosity, hypertelorism, and bifid uvula or cleft palate. In addition cardiac malformations such as atrial septal defect, bicuspid aorta etc. can be seen in this syndrome (Loeys BL et al., 2005) As mentioned above, our patient had no aortic/ arterial abnormalities and hypertelorism. Coexistence of bifid uvula and cardiac manifestations are may be a variant of LDS or incidental.

Informed Consent: Written informed consent was obtained from the patient who participated in this case. Peer-review: Externally peer-reviewed.

Author Contributions: Concept - ZYG; Design- TI; Supervision- OB; Data Collection and/or ProcessingAK; Analysis and/or Interpretation - ZYG; Literature Review -OB; Writing -ZYG; Critical Review -EA

Conflict of Interest: No conflict of interest was declared by the authors.

Financial Disclosure: The authors declared that this study hasn't received no financial support.

\section{References}

Loeys BL, Chen J, Neptune ER et al. A syndrome of altered cardiovascular, craniofacial, neurocognitive and skeletal development caused by mutations in TGFBR 1 or TGFBR2. Nat Genet.2005; 37: 275-81

Loeys BL, Schwarze U, Holm T et al., Aneurysm syndromes caused by mutations in the TGF- $\beta$ receptor. N Engl J Med, 2006; 355: 788- 98.

MacCarrick G, Black JH, Bowdin S et al. LoeysDietz syndrome: a primer for diagnosis and management. Genet Med, 2014; 16: 576-87.

Williams JA, Loeys BL, Nwakanma LU et al., Early surgical experience with Loeys- Dietz: A new syndrome of aggressive thoracic aortic aneurysm disease. Ann Thorac Surg, 2007; 83: $757-63$. 